\title{
Creación de un Currículo de Competencias en Cariología para Estudiantes de Pregrado de Odontología en Chile.
}

\section{Development of a Competency-based Curriculum in Cariology for Undergraduate Dental Students in Chile}

Rodrigo A. Giacaman ${ }^{1 *}$, Vicente Aranguiz², Juliana Botelho1, Ivonne P. Bustos ${ }^{1}$, Rodrigo Cabello ${ }^{3}$, Yuliana Candiales $^{3}$, Ana María Carrizo ${ }^{4}$, Claudia Carvajal ${ }^{5}$, Ramiro Castro ${ }^{1}$, René Castro ${ }^{6}$, Jaime Díaz ${ }^{7}$, Marco DíazYokens $^{8}$, Mario Díaz-Dosque ${ }^{3}$, Natalia Diaz-Garrido', Gerardo Durán ${ }^{9}$, Simone Faleiros ${ }^{3}$, Mario Flores ${ }^{8}$, Juan Fonseca $^{8}$, Margherita Fontana ${ }^{10}$, Manuel Gajardo ${ }^{11}$, Marianela Gómez ${ }^{12}$, Carlos Gonzalez-Cabezas ${ }^{10}$, Sergio González $^{13}$, Roberto Irribarra ${ }^{14}$, Gisela Jara ${ }^{5}$, Soraya León ${ }^{1}$, María Loreto Marró ${ }^{2}$, Claudia Mautz ${ }^{15}$, Alexandra Mustakis $^{16}$, Juan Eduardo Onetto ${ }^{12}$, María Eugenia Ortiz ${ }^{15}$, Darwin Pérez ${ }^{17}$, Vidal Pérez ${ }^{1}$, Valeria Ramírez ${ }^{2}$, Gonzalo Rodríguez ${ }^{3}$, Valeria Rojas ${ }^{6}$, Begoña Ruiz ${ }^{3}$, Alfredo Silva ${ }^{11}$, Iván Urzúa ${ }^{18}$, Carolina del Valle ${ }^{5}$, Carlos Zaror $^{7}$, Gustavo Moncada².

1. Universidad de Talca, Chile

2. Universidad de Los Andes, Chile

3. Universidad de Chile, Chile

4. Universidad de Antofagasta, Chile

5. Ministerio de Salud, Chile

6. Universidad Finis Terrae, Chile

7. Universidad de La Frontera, Chile

8. Universidad San Sebastián, Chile

9. Universidad Arturo Prat, Chile

10. University of Michigan, USA

11. Universidad Andrés Bello, Chile

12. Universidad de Valparaíso, Chile

13. Universidad Mayor, Chile

14. Colegio de Dentistas de Chile, Chile

15. Universidad Austral de Chile, Chile

16. Universidad Católica de Chile, Chile

17. Universidad de La Serena, Chile

18. Universidad Del Desarrollo, Chile

${ }^{*}$ Correspondencia autor: Rodrigo A. Giacaman Universidad de Talca, 2 Norte 685, Talca. |

Teléfono: +56-71-2201546 | Email: giacaman@ utalca.cl

Trabajo recibido el 07/12/2017. Aprobado para su publicación el 10/02/2018

\section{RESUMEN}

Objetivo: Creación de un currículo de competencias mínimas en Cariología, para la formación de los Cirujano-Dentistas egresados de las escuelas de Odontología de Chile. Metodologías: A partir de una reunión de académicos de las Universidades de Talca y de Chile (año 2011), se elaboró una propuesta de currículo inicial, basado en los dominios propuestos por la Unión Europea (Schulte AG y cols). Durante el año 2016, dicha propuesta fue analizada mediante diálogos digitales y grupos de trabajo, con la participación del $96 \%$ de las Escuelas de Odontología existentes en el país, que concluyeron en un documento intermedio. Este documento fue analizado, discutido y perfeccionado durante el Taller para el Desarrollo de un Currículo de Competencias Mínimas en Cariología para las Escuelas de Odontología Chilenas (22/Mayo/2017, Talca, organizado por la Universidad de Talca y la Universidad de Chile) con la asistencia de representantes del $96 \%$ de las escuelas dentales chilenas, Ministerio de Salud de Chile, Colegio de Cirujano-Dentistas de Chile y con la asesoría de los profesores de Cariología Dres. Margherita Fontana y Carlos González-Cabezas (Universidad de Michigan, Ann Arbor, EEUU). Cada grupo de trabajo revisó el documento y envió nuevos comentarios, los que fueron incorporados en el documento final por una comisión asesora.

Resultados: El documento del Currículo en Cariología se organizó en 5 Dominios: 1. Conocimientos base; 2. Determinación de Riesgo, diagnóstico de caries y detección de lesiones de caries; 3 . Toma de decisiones y manejo preventivo no operatorio; 4. Toma de decisiones y manejo operatorio y 5 . Cariología basada en la evidencia, en la práctica clínica y de salud pública. Se consensuaron las definiciones operacionales, las competencias principales y las sub-competencias para cada uno de los dominios. Las sub-competencias fueron clasificadas en tres niveles: A: Ser competente en; B: Tener conocimientos sobre y C: Estar familiarizado con. El documento final fue enviado a todos los participantes del taller para su aprobación y difusión en cada una de las instituciones involucradas.

Conclusiones: Se logró, por medio de consenso, la construcción del Currículo de Competencias mínimas en Cariología para estudiantes de pregrado de Odontología en las universidades chilenas.

PALABRAS CLAVE

Educación, Cariología, Educación en odontología, Caries dental.

Rev. Clin. Periodoncia Implantol. Rehabil. Oral Vol. 11(1); 58-68, 2018.

\section{ABSTRACT}

Objective: Development of a minimum set of competencies in Cariology that every dentist graduated from a Dental School in Chile must have.

Methodology: Starting from a meeting of scholars from the Universities of Talca and Chile (year 2011), an initial proposal for a curriculum was developed, based on the domains proposed by the European Cariology Curriculum (Schulte, et al, 2011). During 2016, this proposal was discussed through online dialogues and working groups, 
with the participation of $95.2 \%$ of the Chilean dental schools, which resulted in an intermediate document. This document was analyzed, discussed and refined during the Workshop for the Development of a Curriculum of Minimum Competencies in Cariology for Chilean Dental Schools (May 22, 2017, Talca, organized by the Universities of Talca and Chile) with the attendance of representatives from $95.2 \%$ of the Chilean dental schools, the Chilean Ministry of Health, the Chilean College of Dentists and with the assistance of the professors of Cariology Margherita Fontana and Carlos GonzálezCabezas (University of Michigan, Ann Arbor, USA). Each working group revised the document and provided feedback, which was incorporated in the final document by an advisory committee, elected on the day of the workshop, including the authors of the present article.

Results: The Cariology Curriculum was organized in 5 Domains: 1. Basic knowledge; 2. Risk assessment, caries diagnosis and caries lesion detection; 3. Decision-making and non-operative preventive treatment; 4 . Decision-making and operative treatment; and 5. Evidence-based, clinical and public health practice. Operational definitions, main competencies and sub-competencies for each domain were agreed. Sub-competencies were classified into three levels: A: Be competent in; B: Have knowledge about, and C: Be familiar with. The final document was sent to all the participants of the workshop for dissemination in each of the institutions involved.

Conclusions: The development of the Competency-based Curriculum in Cariology for undergraduate dental students at Chilean universities was achieved through consensus. KEYWORDS

Education, Cariology, Dental education, Dental caries.

Rev. Clin. Periodoncia Implantol. Rehabil. Oral Vol. 11(1); 58-68, 2018.

\section{INTRODUCCIÓN}

La caries dental es la enfermedad no transmisible más común del ser humano. Según los datos más actualizados, el $35 \%$ de la población a nivel mundial presenta lesiones de caries no tratadas y en Latinoamérica este número es cercano al $45 \%{ }^{(1)}$. La caries afecta fuertemente la calidad de vida, especialmente la autoestima y las relaciones interpersonales, mediante el impacto que tiene en la función masticatoria y estética ${ }^{(2-4)}$

A pesar del creciente número de odontólogos formados en las universidades y al desarrollo de tecnología asociada al tratamiento restaurador, los datos muestran que en los últimos 20 años los indicadores de caries se han mantenido y en algunos casos incluso han aumentado(1). La interpretación de la etiopatogenia de la enfermedad ha sufrido cambios fundamentales en los últimos años, pasando de la visión de una enfermedad infecciosa y transmisible causada por una sola especie bacteriana, a una visión de enfermedad ecológica no transmisible causada por el desequilibrio del biofilm dental como consecuencia del consumo frecuente de azúcares ${ }^{(5-7)}$. Adicionalmente, la formación del odontólogo requiere de la incorporación de herramientas para comprender el rol de factores socioculturales en el desarrollo de la enfermedad y la importancia que durante el manejo integral de los pacientes se incorporen todos estos conceptos $^{(8)}$. En este contexto, la formación del nuevo odontólogo exige una cuidadosa revisión de las competencias profesionales declaradas en su plan de estudios. El Cirujano Dentista egresado de las universidades del país debe ser protagonista activo en lograr cambios del perfil epidemiológico de la caries dental en Chile y de fomentar y entregar salud bucal a nivel individual y comunitario, utilizando la más reciente evidencia científica disponible en cada momento. En particular, la Cariología ha sido el eje central del quehacer del odontólogo desde sus orígenes y por ello su enseñanza y abordaje curricular en las universidades que forman profesionales odontólogos es de central importancia. El estudio de la caries dental es transversal a todas las disciplinas y especialidades de la odontología. Por lo anterior, el contar con odontólogos con un perfil de competencias similares en el país asegurará que la atención de salud bucal y las políticas públicas se alineen para el logro de los objetivos sanitarios de Chile que contribuyan así a mejorar la calidad de vida de los chilenos.

En la enseñanza de la Odontología, se hace necesario estar preparados para introducir nuevos conocimientos durante el aprendizaje del futuro odontólogo y, a su vez, se deben adaptar los conceptos educativos frente a las necesidades dinámicas de los pacientes ${ }^{(9)}$. Con esta base, surge como obligación adaptar y ejecutar, en la organización curricular, la comprensión y aplicación de las bases de la etiopatogenia, prevención y manejo de la enfermedad de caries dental, tanto como los trastornos vinculados con otras patologías dentarias, que entreguen la educación oportuna y actualizada de la enfermedad ${ }^{(10)}$. Se han planteado diversas propuestas para optimizar la enseñanza de la Cariología durante los últimos años. La Unión Europea, basada en los acuerdos de Bolonia, implementó un currículo de contenidos únicos para la educación odontológica en sus 48 países $^{(10,11)}$. Por otra parte, la enseñanza de la
Cariología en EE. UU. y en Japón muestra contenidos parcialmente logrados y con diversos grados de profundidad en el pregrado de sus escuelas dentales ${ }^{(12-14)}$. En un estudio realizado en algunas escuelas de odontología de América Latina, la Cariología es descrita como parte de los currículos, pero muestra alta variabilidad en términos de la planificación y logros alcanzados ${ }^{(15)}$. En Chile, el primer estudio sobre la enseñanza en Cariología, concluye que solo la mitad de las escuelas dentales presentan una adecuada implementación, sin alineación de contenidos teóricos con la práctica clínica y con bajo reconocimiento de los tratamientos no invasivos ${ }^{(16)}$. El segundo y último estudio en Chile muestra que la actual enseñanza de la Cariología en las escuelas dentales es alta en contenidos teóricos, los que mayoritariamente no se imparten a través de prácticas de laboratorio y adicionalmente, la aplicación clínica de los contenidos es escasa(17).

En general, la educación de pregrado en Cariología muestra variadas estrategias metodológicas en el proceso de enseñanza-aprendizaje, siendo necesario trabajar en su reformulación y alcanzar optimizaciones en los criterios aplicados para entrenar a profesores y estudiantes ${ }^{(18)}$. Frente a esta realidad, se propone como objetivo, la creación de un currículo de competencias mínimas en Cariología, para la formación de los Cirujano-Dentistas egresados de las escuelas dentales de pregrado de Chile.

\section{MATERIAL Y MÉTODOS}

La propuesta de currículo mínimo de competencias en Cariología para Chile significó 6 años de trabajo, separados en seis etapas. La primera fue la revisión de las competencias en Cariología, a partir de la propuesta de currículo mínimo realizada por ORCA (European Organisation for Caries Research)(10) y replicado posteriormente en EE.UU.(12), para consensuar un marco común de competencias en Cariología y paralelamente el reconocimiento de la realidad epidemiológica chilena ${ }^{(19-22)}$. Ese trabajo, desarrollado por académicos de la Universidad de Talca y la Universidad de Chile durante el año 2011 , incluyó la adaptación idiomática y el análisis de la pertinencia académica y cultural a la realidad chilena. Entre el año 2011 y el 2017 se llevaron a cabo las demás etapas. La segunda etapa fue la preparación de la propuesta base. En la tercera etapa, este documento fue compartido a los 21 Decanos de las 21 universidades del país que impartían la carrera de Odontología a esa fecha, además del Ministerio de Salud de Chile (MINSAL) y el Colegio de Cirujano Dentistas de Chile y se les encomendó la tarea de nominar a un representante por cada Universidad, para que participara en la revisión de la propuesta. En la cuarta etapa, se recibieron los aportes, observaciones y comentarios de 20 de las 21 universidades (96\%), del MINSAL y del Colegio de Cirujano Dentistas. En la quinta etapa la propuesta fue discutida en un taller especialmente organizado, en el marco del 3er Encuentro Internacional de Cariología, realizado en la Universidad de Talca. (Talca, 22 de mayo 2017). El taller fue acompañado por los profesores Margherita Fontana y Carlos González-Cabezas de la Universidad de Michigan, EE. UU., quienes fueron parte fundamental de un trabajo similar en el mismo país, el 
año 2015. Recogidas las observaciones y propuestas de los participantes en el taller, se concretó la sexta y última etapa que acogió las propuestas y generó el documento final de consenso, en un trabajo efectuado por una Comisión Asesora de 5 personas, nominada unánimemente por los participantes del taller. La propuesta final de Currículo Mínimo basado en Competencias en Cariología para Chile se consensua como la base para que las distintas universidades implementen los cambios necesarios en cada uno de sus proyectos de formación, apoyadas por la Comisión Asesora. Finalmente, este Currículo de Competencias en Cariología para Estudiantes de Pregrado de Odontología en Chile fue presentado el 7 de septiembre de 2017 ante la Asociación Chilena para la Enseñanza de la Odontología (ACHEO), órgano colegiado que coordinará los esfuerzos para la implementación en el país.

\section{RESULTADOS}

Estructura y conceptos contenidos en la propuesta.

El documento final fue estructurado en base a 5 dominios principales, cada uno de ellos con una o más competencias principales y subcompetencias:

Dominio 1: El conocimiento base.

Dominio 2: Determinación de riesgo, diagnóstico de caries y detección de lesiones de caries.

Dominio 3: Toma de decisiones y manejo preventivo no operatorio.

Dominio 4: Toma de decisiones y manejo operatorio.

Dominio 5: Cariología basada en la evidencia en la práctica clínica y de salud pública.

Para la definición del nivel de logro de las competencias, se adoptó la propuesta del perfil y competencias para el dentista europeo ${ }^{(23)}$. En donde las competencias pueden ser exigidas en tres niveles de desarrollo: "ser competente en", "tener conocimiento sobre" o "estar familiarizado con", basado en las siguientes definiciones operacionales para cada nivel de logro:

Ser competente: el Cirujano Dentista (CIRUJANO DENTISTA) al momento de su titulación debe demostrar conocimiento teórico y comprensión del aspecto a lograr, junto con una adecuada experiencia clínica que le permitan resolver los problemas encontrados de manera independiente o sin asistencia.

Tener conocimiento sobre: el CIRUJANO DENTISTA debe demostrar sólido conocimiento teórico y comprensión del tema, pero puede tener solo una limitada experiencia clínica o práctica.

Estar familiarizado con: EI CIRUJANO DENTISTA debe demostrar comprensión básica del aspecto a lograr, pero no requiere tener experiencia clínica o llevar a cabo procedimientos de manera independiente.

La descripción de los dominios, competencias, sub-competencias y nivel de logro del Currículo en Cariología para las universidades chilenas se detalla a continuación:

\section{FUENTES DE APOYO FINANCIERO}

Sin fuentes de apoyo financiero

\section{CONFLICTO DE INTERESES}

Los autores declaran no tener ningún conflicto de intereses.

Dominios con principales competencias de apoyo

\section{Dominio 1: El Conocimiento Base}

Este dominio describe los conocimientos básicos necesarios para lograr las competencias declaradas en los dominios 2 a 5. Para alcanzar las competencias necesarias se requieren distintas profundidades en el conocimiento de cada uno de los aspectos del conocimiento de base.

\section{Competencia Principal}

Al graduarse, el CIRUJANO DENTISTA debe ser competente en la toma de decisiones para realizar el diagnóstico, prevención y tratamiento de la enfermedad caries y de otras lesiones no cariosas en individuos y comunidades, mediante la aplicación del conocimiento y la comprensión de las ciencias biológicas, médicas, básicas y sociales, basado en la mejor evidencia disponible.

\section{Subcompetencias}

a.Con respecto al desarrollo, crecimiento y estructura de los tejidos orales relevantes, el CIRUJANO DENTISTA egresado debe:

\begin{tabular}{l|l|l}
\hline Ser competente en: & Tener conocimiento sobre: & Estar familiarizado con: \\
\hline $\begin{array}{l}\text { Evaluar el estado de desarrollo, crecimiento y es- } \\
\text { tructura de los tejidos orales relevantes. }\end{array}$ & $\begin{array}{l}\text { Desarrollo normal, crecimiento y estructura de } \\
\text { tejidos dentales y orales (Ejemplo, tejidos duros } \\
\text { dentales, pulpa y glándulas salivales) a nivel ma- } \\
\text { croscópico, microscópico y molecular. } \\
\text { Trastornos del desarrollo de tejidos dentales y y } \\
\text { prales a nivel macroscópico, microscópico y mo- } \\
\text { jicos trastornos del desarrollo de dichos te- }\end{array}$ & \\
& lecular. & \\
\hline
\end{tabular}

Subcompetencias

b. Con respecto a la etiología, patogénesis y factores que modifican la caries dental y otros trastornos de los tejidos duros dentales, el CIRUJANO DENTISTA egresado debe:

\begin{tabular}{l|l|l}
\hline Ser competente en: & Tener conocimiento sobre: & Estar familiarizado con: \\
\hline Describir la microbiología del medioambiente bu- & Eventos bioquímicos en el biofilm, en saliva y en & \\
cal en salud y la asociada al proceso de caries & los tejidos duros dentales. & \\
en sus distintos estadios. En este punto deberá & El rol del biofilm dental en la saliva, sus propieda- & \\
incluir las actividades metabólicas de especies & des tamponadoras y los efectos de la saturación & \\
individuales, así como las relaciones antagónicas & de la misma. & \\
o simbióticas implicadas entre diversas especies & El rol de los factores medioambientales, dieta, \\
del biofilm dental. & fármacos, enfermedades sistémicas relacionadas & \\
Discutir los mecanismos y procesos dinámicos & con la caries y otros condicionantes relacionados & \\
involucrados en el mantenimiento del estado de & al hospedero. & \\
salud, así como la respuesta del huésped en ca- & El rol de los fluoruros y sus beneficios. & \\
ries y erosión a un nivel macroscópico, microscó- & El rol de los determinantes sociales de la salud & \\
pico y molecular. & relacionados con la caries y otras lesiones no ca- & \\
& riosas & \\
\hline
\end{tabular}

60 | Rev. Clin. Periodoncia Implantol. Rehabil. Oral Vol. 11(1); 58-68, 2018. 
Dominio 1: El Conocimiento Base (continuación)

Subcompetencias

c. Con respecto a la detección, evaluación y diagnóstico, el CIRUJANO DENTISTA egresado debe:

\begin{tabular}{l|l|}
\hline Ser competente en: & Tener conocimiento sobre: \\
\hline & $\begin{array}{l}\text { Bases físicas y biológicas de los cambios en los } \\
\text { tejidos duros dentales, relacionados con la detec- } \\
\text { ción, evaluación de lesiones caries y otras lesio- } \\
\text { nes no cariosas } \\
\text { La imagenología como complemento a la detec- } \\
\text { ción y evaluación de caries y otras lesiones no } \\
\text { cariosas } \\
\text { Evaluar los resultados de los métodos diagnósti- } \\
\text { cos aplicados a lesiones de caries y otros trastor- } \\
\text { nos de los tejidos duros dentales, desde el punto } \\
\text { de vista individual y comunitario. }\end{array}$ \\
\hline
\end{tabular}

Estar familiarizado con:

El modo de acción y las limitaciones de los métodos emergentes para la detección, evaluación, diagnóstico de caries y otras lesiones no cariosas

Subcompetencias

d. Con respecto a las ciencias del comportamiento, el Cirujano Dentista egresado debe:

\begin{tabular}{|c|c|c|}
\hline Ser competente en: & Tener conocimiento sobre: & Estar familiarizado con: \\
\hline & $\begin{array}{l}\text { Principios básicos de ética, bioética y profesiona- } \\
\text { lismo, enfocados en la realidad sociocultural de } \\
\text { cada población a intervenir. }\end{array}$ & $\begin{array}{l}\text { Las ciencias del comportamiento, incluidos los } \\
\text { principios psicológicos, sociológicos y de comuni- } \\
\text { cación que promuevan los cambios de conducta } \\
\text { en salud por parte del individuo/cuidador y su co- } \\
\text { munidad. }\end{array}$ \\
\hline
\end{tabular}

\section{Subcompetencias}

e. Con respecto a la prevención individual y comunitaria, el Cirujano Dentista egresado debe:

\begin{tabular}{|c|c|}
\hline Ser competente en: & Tener conocimiento sobre: \\
\hline & $\begin{array}{l}\text { El modo de acción, composición, propiedades, } \\
\text { limitaciones y efectos secundarios o adver- } \\
\text { sos a nivel general y local (pulpa, dentina, es- } \\
\text { malte), de los materiales de uso odontológico, } \\
\text { productos y técnicas comúnmente disponibles } \\
\text { para el manejo de la caries dental y otras lesio- } \\
\text { nes no cariosas, a nivel individual y comunitario. } \\
\text { Relación costo/beneficio de materiales a utilizar en } \\
\text { el manejo preventivo no quirúrgico y quirúrgico de } \\
\text { la caries dental y otras lesiones no cariosas. }\end{array}$ \\
\hline
\end{tabular}

Estar familiarizado con:

Las bases teóricas de las estrategias individuales y comunitarias emergentes.

Los materiales para la prevención y el manejo

de la caries dental y otros trastornos de los

tejidos duros dentales.

\section{Subcompetencias}

f. Con respecto a la epidemiología y metodología de la investigación, el Cirujano Dentista egresado debe:

\begin{tabular}{|c|c|c|}
\hline Ser competente en: & Tener conocimiento sobre: & Estar familiarizado con: \\
\hline & $\begin{array}{l}\text { Perfil epidemiológico de caries a nivel nacional e } \\
\text { internacional. } \\
\text { Los principios de la evaluación de riesgo y las } \\
\text { herramientas existentes para determinar el } \\
\text { riesgo cariogénico. }\end{array}$ & $\begin{array}{l}\text { Las bases de la epidemiología. } \\
\text { Las metodologías de investigación y sus limita- } \\
\text { ciones, incluyendo diseño de estudio, muestreo, } \\
\text { sesgo y estadística, para la interpretación de la } \\
\text { evidencia disponible. }\end{array}$ \\
\hline
\end{tabular}


Dominio 2: Determinación de riesgo, diagnóstico de caries y detección de lesiones de caries.

Este dominio representa un puente común entre el conocimiento básico (o de base) y la evaluación de la necesidad de manejo no operatorio y operatorio para la prevención y el control de lesiones de caries. Las competencias en la toma de decisiones clínicas son necesarias para resolver apropiadamente, basado en evidencia científica los problemas de la clínica y la práctica de la salud pública.

\section{Determinación de riesgo}

Competencia principal

Clasificar el nivel de riesgo de un paciente odontológico basado en conocimiento científico, e identificar y estimar la probabilidad de desarrollar nuevas lesiones o la progresión de lesiones existentes durante un periodo de tiempo específico.

\section{Subcompetencias}

El Cirujano Dentista egresado debe:

\begin{tabular}{l}
\hline Ser competente en: \\
\hline Obtener la información, identificando los indicado- \\
res de riesgo más relevantes para el paciente consi- \\
derando el rol del biofilm oral, de la dieta, la saliva, \\
fluoruros, factores socioculturales y otros factores \\
relacionados con caries u otras patologías no cario- \\
sas de los tejidos duros. \\
Analizar los principales factores de riesgo cariogénico, \\
que incluyen: historia médica, oral, índices de lesio- \\
nes de caries, social y económica. Hábitos de salud \\
oral, incluyendo la higiene oral, conocimientos, prefe- \\
rencias y medidas de autocuidado, hábitos de dieta \\
(familiares, comunitarios y socioculturales) y factores \\
biológicos intraorales, experiencia de caries, uso de \\
fluoruros y salud sistémica considerando nuevos fac- \\
tores validados, así como nueva evidencia emergente. \\
Comunicar los resultados de la evaluación del ries- \\
go al paciente u otros entregando recomendaciones \\
que le permitan reducir el riesgo de desarrollar nue- \\
vas lesiones de caries o la progresión de lesiones \\
existentes. \\
Reevaluar el riesgo cariogénico del paciente para \\
así reforzar o modificar las medidas que no hayan \\
tenido buen resultado o para reforzar las acciones \\
que el paciente ha llevado a cabo.
\end{tabular}
Estar familiarizado con:

Conceptos y técnicas de manejo sociológico y psicológicoefectivodelarelaciónpaciente-dentista. Valoración de la información emergente relacionada con factores o indicadores de riesgo, junto con la ponderación objetiva del riesgo cariogénico.

b. Diagnóstico de caries como enfermedad Competencia principal

El egresado será competente en la recolección, análisis e integración de datos de signos y síntomas de caries dental, y de conductas y circunstancias en que la persona vive y se desarrolla para realizar el diagnóstico de la enfermedad.

Subcompetencias

El Cirujano Dentista egresado debe:

\begin{tabular}{l|l|l}
\hline Ser competente en: & Tener conocimiento sobre: & Estar familiarizado con: \\
\hline Integrar y sintetizar la información obtenida para & & \\
lograr un diagnóstico integral y luego el diagnós- & & \\
tico de caries del paciente. & & \\
Evaluar la influencia de los valores, preferencias & & \\
y conductas de la persona considerando su con- & & \\
texto sociocultural. & & \\
\hline
\end{tabular}

c. Detección de lesiones de caries

Competencia principal

EI CIRUJANO DENTISTA egresado será competente en la identificación y evaluación del estado de la estructura dental afectada y del nivel de actividad de una lesión sobre la superficie del diente.

\section{Subcompetencias}

El Cirujano Dentista egresado debe:

\begin{tabular}{l|l|l}
\hline Ser competente en: & Tener conocimiento sobre: & Estar familiarizado con: \\
\hline $\begin{array}{l}\text { Reconocer tejido dentario anormal y diferenciar } \\
\text { entre tejidos duros con lesión de caries y sin le- }\end{array}$ & $\begin{array}{l}\text { La evaluación de nuevos métodos para detección } \\
\text { y clasificación del proceso de caries que contribu- }\end{array}$ & \\
nocer lesiones primarias y secundarias tanto a & $\begin{array}{l}\text { yen al diagnóstico de la enfermedad. } \\
\text { La relación costo/beneficio de los nuevos méto- } \\
\text { nivel coronario como radicular. }\end{array}$ & \\
$\begin{array}{l}\text { Valorar las distintas etapas del proceso de caries, } \\
\text { según grado de severidad y tejidos duros afec- } \\
\text { de caries según atingencia a la población obje- } \\
\text { tados, tanto en los signos como en los síntomas }\end{array}$ & & \\
relacionados con caries. & & \\
$\begin{array}{l}\text { Evaluar el grado de actividad y progresión de las } \\
\text { diferentes etapas del proceso de caries. }\end{array}$ & & \\
Efectuar diagnóstico diferencial entre lesiones & & \\
cariosas y lesiones no cariosas. & & \\
\hline
\end{tabular}




\section{Dominio 3: Toma de decisiones y manejo preventivo no operatorio}

Este domino dice relación con el manejo de la caries y otras lesiones no cariosas, con énfasis en la planificación del control y cuidado preventivo en el

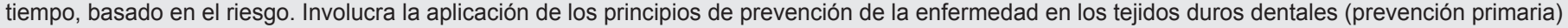

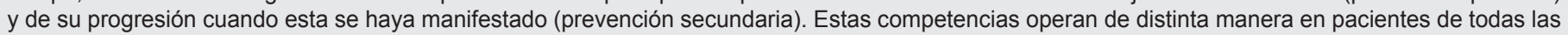
edades. Los objetivos de control y prevención debieran estar claramente definidos para poder evaluar los resultados.

a. Comunicación con el paciente, familia y comunidad en diferentes ambientes relacionados con la salud.

\section{Competencia Principal}

El Cirujano Dentista egresado debe ser competente en comunicar los aspectos de promoción de la salud, prevención y control de manera efectiva, inte-

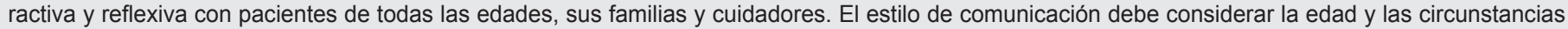

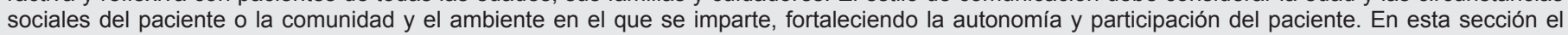
término "paciente" se refiere a las familias y cuidadores cuando sea pertinente.

\section{Subcompetencias}

El Cirujano dentista egresado debe:

\begin{tabular}{|c|c|c|}
\hline Ser competente en: & Tener conocimiento sobre: & Estar familiarizado con: \\
\hline
\end{tabular}




\section{Dominio 3: Toma de decisiones y manejo preventivo no operatorio (continuación)}

a. Toma de decisiones clínicas preventivas conducentes a terapias preventivas no operatorias.

\section{Competencia Principal}

El Cirujano Dentista egresado debe ser competente en recolectar, interpretar y sintetizar toda la información relevante, contenida en la ficha clínica, necesaria para formular adecuadas opciones de tratamiento que puedan ser presentadas y consensuadas con el paciente para llegar a decisiones respecto del plan de tratamiento individualizado. Esto incluye una estrategia de control y cuidados preventivos en el tiempo, acorde a las necesidades, riesgos y capacidad de cumplimiento de los individuos, sus familias, grupos y comunidades. Este manejo no quirúrgico de la enfermedad debiera considerar no solamente el sitio y el diente sino también factores relacionados con el paciente. Además de evaluar sistemáticamente todos los posibles desenlaces del tratamiento preventivo en cada cita de control y formular planes de tratamiento alternativo cuando sea necesario.

Subcompetencias

El Cirujano Dentista egresado debe:

\begin{tabular}{|c|c|c|}
\hline Ser competente en: & Tener conocimiento sobre: & Estar familiarizado con: \\
\hline
\end{tabular}




\section{Dominio 4: Toma de decisiones y manejo operatorio.}

Este dominio se ocupa del manejo de la lesión de caries y otras alteraciones de los tejidos dentales, con énfasis en la planificación y mantenimiento del tratamiento restaurador, acompañado de atención preventiva continua (Dominio 3). Involucra aplicar los principios de la preservación de los tejidos duros del diente y de la salud del órgano pulpar. Además, está alineado con los fundamentos clínicos de la odontología restauradora, endodoncia, ortodoncia, periodoncia y prótesis, en cuanto a la ejecución de la fase restauradora o un plan de tratamiento integral. Reconoce la intervención operatoria de lesiones cavitadas, valorando la actividad de la lesión de caries.

a.Toma de decisiones clínicas que conducen al manejo operatorio.

EI CIRUJANO DENTISTA egresado, debe ser competente en el diagnóstico (recolección, interpretación y síntesis) de toda la información pertinente necesaria para formular las diferentes opciones de tratamiento individualizado y discutir estas con el paciente, para llegar a decisiones compartidas en la implementación del plan de tratamiento definido. Esto requiere la capacidad de decidir cuándo es apropiado intervenir de manera operatoria, lesiones de caries cavitadas y saber cómo hacerlo, así como otras lesiones no cariosas, comprendiendo las consecuencias y pronóstico de las decisiones tomadas.

\section{Subcompetencias}

El Cirujano Dentista egresado debe:

\begin{tabular}{l|l|l}
\hline Ser competente en: & Tener conocimiento sobre: & Estar familiarizado con: \\
\hline $\begin{array}{l}\text { Seleccionar la opción de tratamiento apropiada } \\
\text { para cada situación, basada en conocimientos } \\
\text { sólidos de toda la gama de opciones de trata- } \\
\text { miento no operatorio y operatorio disponibles. } \\
\begin{array}{l}\text { Reconocer, comprender y manejar las conse- } \\
\text { cuencias de la intervención operatoria. }\end{array}\end{array}$ & $\begin{array}{l}\text { Las reaciones del complejo pulpodentinario ante } \\
\text { el proceso de la caries y a los diferentes procedi- } \\
\text { Leflexionar continuamente sobre el proceso de } \\
\text { de restauraciones. }\end{array}$ & \\
$\begin{array}{l}\text { toma de decisiones y los resultados de la inter- } \\
\text { vención operatoria en Cariología. }\end{array}$ & & \\
\hline $\begin{array}{l}\text { b.Manejo Operatorio } \\
\text { El Cirujano Dentista egresado debe ser competente para llevar a cabo el tratamiento operatorio apropiado de las lesiones de caries, mientras preserva el } \\
\text { máximo de la estructura dental y la salud pulpar, en el contexto del plan de tratamiento integral del paciente. Además, debe restaurar la pérdida de tejido } \\
\text { duro dental tanto en forma, función y estética y al mismo tiempo, restablecer y promover la salud oral }\end{array}$ & \\
\hline
\end{tabular}

\section{Subcompetencias}

El Cirujano Dentista egresado debe:

\begin{tabular}{l}
\hline Ser competente en: \\
\hline Decidir cuándo, cómo y en qué medida debe eli- \\
minar el tejido dentario cariado, antes de la co- \\
locación de una restauración, teniendo en cuen- \\
ta la capacidad del diente de ser restaurado, la \\
preservación de la estructura dental y la vitalidad \\
del órgano pulpar, en coherencia con el plan de \\
tratamiento integral del paciente. \\
La selección del instrumental y en el manejo de \\
los biomateriales restauradores apropiados, con- \\
siderando sus propiedades físicas y químicas, \\
biocompatibilidad y longevidad. \\
Seleccionar y realizar las técnicas operatorias \\
apropiadas, tanto para el biomaterial como para \\
el caso.
\end{tabular}

Tener conocimiento sobre:

El impacto de los procedimientos restaurativos sobre la mucosa, los tejidos periodontales, la oclusión y la función oral.

El comportamiento biomecánico de las restauraciones.

c.Mantención del paciente

Competencia Principal

El Cirujano Dentista egresado debe ser competente en la reevaluación de los factores de riesgo (Dominio 2), como contexto de la evaluación del éxito de un tratamiento restaurador específico. Además, debe decidir el esquema de mantención del paciente (frecuencia de controles), la reparación o el reemplazo de restauraciones y realizar la instrucción apropiada del paciente, para reducir el daño de éstas.

\section{Subcompetencias}

El Cirujano Dentista egresado debe:

\begin{tabular}{l|l|l}
\hline Ser competente en: & Tener conocimiento sobre: & Estar familiarizado con: \\
\hline $\begin{array}{l}\text { La evaluación y monitoreo de los resultados del } \\
\text { tratamiento a través del tiempo. }\end{array}$ & & $\begin{array}{l}\text { Los aspectos económicos de la terapia restaura- } \\
\text { dora (aspectos sanitarios; costo-beneficio) }\end{array}$ \\
para la correcta estimación del estado de la res- \\
tauración (mantención, reparación o recambio). \\
$\begin{array}{l}\text { Establecer un esquema de mantenimiento de } \\
\text { restauraciones, para prolongar la longevidad de } \\
\text { estas }\end{array}$ & & \\
\hline
\end{tabular}

\section{Estar familiarizado con:}

Alternativas con mayor evidencia de nuevos métodos para la eliminación de caries, lo que comprende tanto la identificación y detección de lo que debe eliminarse, así como el procedimiento clínico de eliminación del tejido dental cariado. Alternativas de técnicas y biomateriales restauradores de mayor evidencia. 
Dominio 5: Cariología basada en la evidencia en la práctica clínica y de salud pública

Este dominio se ocupa de los elementos fundamentales de la práctica odontológica basada en la evidencia dentro del plan de estudios universitarios, que fundamenta la doble faceta de la Cariología clínica (en relación con las personas) y la Cariología en salud pública (en relación a grupos o la comunidad).

a.Cariología en Salud Pública

Competencia Principal

Un Cirujano Dentista debe ser competente en la prevención y el control de la caries dental y otros trastornos de los tejidos duros del diente a nivel individual y comunitario. Esto requiere la comprensión completa de la epidemiología, promoción de la salud y las estrategias preventivas, su integración en los sistemas de cuidado de la salud oral y la interacción con otros trastornos orales, estado general de salud, la nutrición y el contexto socioeconómico.

\section{Subcompetencias}

El Cirujano Dentista egresado debe:

\begin{tabular}{|c|c|c|}
\hline Ser competente en: & Tener conocimiento sobre: & Estar familiarizado con: \\
\hline
\end{tabular}

Cariología basada en evidencia

Competencia Principal

El Cirujano Dentista debe desarrollar práctica basada en la evidencia para aplicarla a los campos de la caries dental, lesiones por malformación, lesiones por erosión, lesiones traumáticas y otros trastornos de los tejidos duros del diente.

\section{Subcompetencias}

El Cirujano Dentista egresado debe:

\begin{tabular}{l|l|l}
\hline Ser competente en: & Tener conocimiento sobre: & Estar familiarizado con: \\
\hline $\begin{array}{l}\text { Buscar y utilizar las guías clínicas vigentes rela- } \\
\text { cionadas con el manejo de la caries. }\end{array}$ & $\begin{array}{l}\text { Los principios de la odontología basada en la evi- } \\
\text { dencia y la jerarquía de la evidencia. }\end{array}$ & \\
El traslado de los resultados de las investigacio- & Los métodos de comunicación de la odontología \\
nes a la práctica de salud tanto clínica como de & basada en la evidencia para las personas, grupos & \\
salud pública relacionadas con el manejo de la la & y poblaciones. & \\
caries. & $\begin{array}{l}\text { Las ventajas y desventajas de las guías clínicas. } \\
\text { Los principios de la investigación, incluyendo el } \\
\text { diseño del estudio, tamaño de muestra, el sesgo } \\
\text { y la bioestadística. }\end{array}$ & \\
\hline
\end{tabular}

\section{DISCUSIÓN}

Dada la situación de caries a nivel global y en particular en Chile(19) resulta urgente abordar la enfermedad desde diversas aristas. En Chile, la caries dental se expresa desde un $16,8 \%$ a los dos años edad hasta un $100 \%$ en la edad adulta. Un estudio realizado en la zona sur del país, en el marco de la construcción del diagnóstico de salud bucal de la población preescolar, concluye que se observan altas prevalencias de caries en edades muy tempranas, que la posición socioeconómica se encuentra asociada a la experiencia de caries y que los hábitos de nutrición se encuentran relacionados con el desarrollo de la enfermedad en todos los niveles socioeconómicos ${ }^{(24)}$. De los pocos estudios epidemiológicos existentes, se advierte una alta prevalencia de caries a los 12 años de edad, con un $62,5 \%$ y un promedio de 1,9 dientes afectados por caries ${ }^{(25)}$. En dicho estudio se observan diferencias estadísticamente significativas de la prevalencia de caries de acuerdo con el nivel socioeconómico. El perfil epidemiológico de caries en Chile supone un complejo desafío, el cual debe ser abordado con el concurso de distintas estrategias. Una de ellas es la manera en que los profesionales identifican el problema, lo previenen, lo planifican e implementan acciones para su control. Este aspecto se relaciona íntimamente con la educación que los futuros profesionales reciben durante su formación en el pregrado. Así, es de la mayor importancia que los futuros odontólogos reciban una educación actualizada y centrada en el logro de competencias que permitan contribuir no solo al manejo clínico de lesiones a personas individuales, sino a la activa participación en el diseño de programas para la promoción de la salud y la prevención efectiva de la enfermedad a nivel de la comunidad y de manera interdisciplinaria, incluso participando en la toma de decisiones en las políticas públicas. Desafortunadamente, la formación 
de los odontólogos actuales en las más de veinte universidades que imparten la carrera de Odontología en Chile es altamente heterogénea y carece de directrices comunes ${ }^{(16,17)}$, para el abordaje de la enfermedad no transmisible más común del ser humano, la más prevalente de las bucales y que constituye la inmensa mayoría del tiempo que dedica el odontólogo durante su ejercicio laboral. Por tanto, este esfuerzo de la odontología chilena de contar con un marco de competencias mínimas comunes para el odontólogo se avizora como un paso sustantivo para la consecución del objetivo de disminuir la alta prevalencia de lesiones de caries en el país, a todas las edades y contextos socioculturales.

La construcción por consenso con amplia y activa participación de los educadores del área $(95,2 \%)$, por un período prolongado de tiempo (6 años) del currículo de competencias mínimas para la enseñanza de la Cariología, permitió reflexionar sobre la forma de unificar criterios para educar a los futuros odontólogos de manera de disminuir la frecuencia y severidad de la enfermedad caries dental en la población chilena. Estos hechos respaldan su validez y se espera que esta decisión dote a Chile de una pauta entre los principales actores de la odontología para la implementación de competencias profesionales esenciales para la formación del dentista. De esta forma, independientemente de su lugar de formación, el CIRUJANO DENTISTA contribuirá a mejorar la salud bucal a nivel individual y poblacional. El proceso se centró en la solución de los problemas del paciente, basado en intervenciones soportadas por la evidencia disponible y tomando en cuenta la pericia del cirujano dentista y las necesidades de tratamiento de los pacientes, destinados a mantener o re-establecer la salud oral y preservar las estructuras dentarias, coincidiendo con lo publicado por los investigadores a cargo de la confección de un marco de competencias en Cariología para la Unión Europea ${ }^{(10)}$. El problema de mayor relevancia que se enfrentó fue la integración de conocimientos anatómicos, histológicos, moleculares, clínicos, de ciencias del comportamiento y de otras ciencias, que soporten los dominios descritos, con foco en el aprendizaje clínico, hecho que ya había sido observado en la propuesta europea(26). Especia acuerdo hubo en la necesidad del desarrollo de competencias sobre el manejo no operatorio en Cariología, estrategias preventivas, métodos para la preservación de la estructura dentaria, éxito en el manejo de la enfermedad y reconocimiento de las tasas de fracaso, tanto como su evaluación a corto y largo plazo. Sobre el manejo operatorio, se destacó la necesidad de una alta habilidad psicomotora, aspecto crítico para e ejercicio profesional, todos antecedentes coincidentes con lo señalado durante esta parte del proceso en Europa ${ }^{(27)}$, durante el trabajo conjunto entre ORCA y la Association for Dental Educacion in Europe (ADEE).

Lesiones hipoplásicas, hipomineralización (opacidades), erosión, lesiones no erosivas, crack dentario, hipersensibilidad dentinaria y otras patologías dentarias fueron finalmente incluidas en el currículo, después de una larga discusión, privilegiando los fundamentos del diagnóstico diferencial, lo que igualmente había sido señalado en la versión europea de este proceso de análisis ${ }^{(28)}$

La discusión sobre las posibilidades de implementar el presente currículo se centró en el desafío que incluye gran diversidad de factores, tales como probables cambios en la prevalencia de la enfermedad, desarrollo de materiales y tecnologías, competencias culturales y de comportamiento y educación interprofesional. Una adecuada instalación de este currículo precisa, en la mayoría de las escuelas de odontología el profundizar habilidades y conocimientos en ciencias básicas, éticas y profesionalismo, responsabilidad social y habilidad para interactuar con otros profesionales. Por lo anterior, la formación del futuro odontólogo requerirá el apropiado logro de las competencias contenidas en el presente documento, pero adicionalmente, las universidades deberán emprender una importante discusión de cómo profundizar el liderazgo de los profesionales que egresarán. Tema que surgió como consecuencia del proceso de construcción del currículo de Cariología en EE.UU.(8).

Durante la discusión, tanto de este documento, como en las instancias previas a él, existió un acuerdo general sobre la necesidad que el currículo desarrolle competencias en la evaluación del riesgo de contraer la enfermedad, de su manejo preventivo y cómo se aplican estos conocimientos a nivel individual y poblacional. La conclusión general fue que la educación se debe convertir en quizás la más importante o la única herramienta para cerrar la brecha existente entre el manejo preventivo precoz y el manejo curativo tardío de la enfermedad y sus secuelas. Se ha reportado que para alcanzar esta meta se debe explicitar la idea de lograr un pensamiento crítico por medio de metodologías de resolución de problemas $^{(8)}$. Fruto de la experiencia en otros países, es posible afirmar que las tareas incluyen revisar la estructura de las escuelas, el entrenamiento de los académicos y alumnos y revisar los estándares de los niveles de atención versus las expectativas de los pacientes.

En conclusión, mediante un consenso sin precedentes en Chile, se logró materializar la construcción del Currículo de Competencias en Cariología para estudiantes de pregrado de las universidades chilenas, con el respaldo de la vasta mayoría de las universidades de Chile que imparten la carrera, representantes del Ministerio de Salud y del Colegio de Cirujano-Dentistas de Chile. La entrega final del documento a la Asociación Chilena para la Enseñanza de la Odontología, refrenda el espíritu de los proponentes de lograr un consenso nacional sobre la necesidad de contar con competencias formativas homogéneas en el país para un abordaje de la caries dental de manera pertinente, que finalmente se materialice en el control de la enfermedad y contribuya al bienestar y la calidad de vida de los chilenos.

\section{RECONOCIMIENTOS}

Las siguientes personas participaron en la adaptación inicial del documento y en la generación del primer borrador del Currículo: Juliana Botelho, Rodrigo Cabello, Ramiro Castro, Mario Díaz, Constanza Echeverría, Simone Faleiros, Rodrigo Giacaman, Patricia Jiménez, Soraya León, Vanesa Pailahual, Gonzalo Rodríguez, Begoña Ruiz e Iván Urzúa.

Las siguientes personas actuaron como coordinadores de los grupos de trabajo durante el taller y fueron responsables del envío de las conclusiones de cada grupo: Natalia Díaz, Iván Urzúa, Vicente Aránguiz, Juan Eduardo Onetto y Rodrigo Cabello.

Todos los autores participaron en la discusión remota y durante el Taller realizado en la Universidad de Talca el 22 de mayo de 2017. Rodrigo Giacaman actuó como coordinador general del trabajo de construcción del Currículo. Gustavo Moncada redactó el primer borrador del presente artículo.

Los autores agradecen a la Srta. Lisbet Yañez de la Escuela de Egresados de la Universidad de Talca, por el trabajo de organización del Taller.

\section{Bibliografía}

1. Kassebaum NJ, Bernabé E, Dahiya M, Bhandari B, Murray CJ, Marcenes W. Global Burden of Untreated Caries: A Systematic Review and Metaregression. J Dent Res 2015; 94: 650-658

2. León S, Bravo-Cavicchioli D, Correa-Beltrán G, Giacaman RA. Validation of the Spanish version of the Oral Health Impact Profile (OHIP-14Sp) in elderly Chileans. BMC Oral Health 2014; 14: 95

3. Martins-Júnior PA, Vieira-Andrade RG, Corrêa-Faria P, Oliveira-Ferreira F, Marques LS, Ramos-Jorge ML. Impact of early childhood caries on the oral health-related quality of life of preschool children and their parents. Caries Res 2013; 47: 211-8 4. Sheiham A. Oral health, general health and quality of life. Bull World Health Organ 2005; 83: 644

5 . Fejerskov $\mathrm{O}$. Changing paradigms in concepts on dental caries: consequences for oral health care. Caries Res 2004; 38: 182-91

6. Simón-Soro A, Mira A. Solving the etiology of dental caries. Trends Microbio 2015; 23: 76-82

7. Giacaman RA. Sugars and beyond. The role of sugars and the other nutrients and their potential impact on caries. Oral Dis. 2017 [Epub ahead of print]

8. Fontana M, Gonzalez-Cabezas C, de Peralta T, Johnsen DC. Dental Education Required for the Changing Health Care Environment. J Dent Educ 2017; 81: eS153 eS161
9. Gerbert B, Badner V, Maguire B, Martinoff J, Wycoff S, Crawford W. Recent graduates' evaluation of their dental school education. J Dent Educ 1987; 51: $697-$ 700

10. Schulte AG, Pitts NB, Huysmans MC, Splieth C, Buchalla W. European core curriculum in cariology for undergraduate dental students. Caries Res 2011; 45: 336-45

11. Plasschaert AJ, Lindh $C$, McLoughlin J, Manogue M, Murtomaa $H$, Nattestad A, Sanz M. Curriculum structure and the European Credit Transfer System for European dental schools: part I. Eur J Dent Educ 2006; 10: 123-30

12. Fontana M, Guzman-Armstrong S, Schenkel AB, Allen KL, Featherstone J Goolsby S, Kanjirath P, Kolker J, Martignon S, Pitts N, Schulte A, Slayton RL, Young D. Wolff M. Development of a Core Curriculum Framework in Cariology for U.S. Dental Schools. J Dent Educ 2016; 80: 705-20

13. Clark TD, Mjor IA. Current teaching of cariology in North American dental schools. Oper Dent 2001; 26: 412-8

14. Fukushima M, Iwaku M, Mjor IA. Cariology in Japanese dental schools. Int Dent J 2004; 54: 269-72

15. Martignon S, Gomez J, Tellez M, Ruiz JA, Marin LM, Rangel MC. Current cariology education in dental schools in Spanish-speaking Latin American countries. J Dent Educ 2013; 77: 1330-7 
16. Diaz-Yokens M, Gonzalez S, Giacaman R, Araya-Bustos M, Moncada G, Martignon S. Enseñanza de cariología en Escuelas Dentales de Chile. En revisión Rev Clin Periodoncia Implantol Rehabil Oral. 2017.

17. Aránguiz V, Marró M, Ramírez Lobos V, Fontana M, Moncada G. Contenidos de cariología impartidos por Escuelas Dentales Chilenas: Estudio transversal. En revisión Rev Clin Periodoncia Implantol Rehabil Oral. 2017.

18. Nascimento MM, Behar-Horenstein LS, Feng X, Guzman-Armstrong S, Fontana M. Exploring How U.S. Dental Schools Teach Removal of Carious Tissues During Cavity Preparations. J Dent Educ 2017; 81: 5-13

19. Ministry of Health (MINSAL). Diagnostic of the oral health situation. Chilean Government 2007; Santiago, Chile. [Consultado 23/08/2015] http://web.minsal.cl/ portal/url/item/7dc33df0bb34ec58e04001011e011c36.pdf

20. Urzua I, Mendoza C, Arteaga O, Rodriguez G, Cabello R, Faleiros S, Carvajal P, Munoz A, Espinoza I, Aranda W, Gamonal J. Dental caries prevalence and tooth loss in chilean adult population: first national dental examination survey. Int J Dent 2012; 2012: 810170

21. Giacaman RA, Bustos IP, Bravo-Leon V, Marino RJ. Impact of rurality on the oral health status of 6-year-old children from central Chile: the EpiMaule study. Rural and Remote Health 2015; 15
22. Quinteros ME, Cáceres DD, Soto A, Mariño RJ, Giacaman RA. Caries experience and use of dental services in rural and urban adults and older adults from central Chile. Int Dent J 2014; 64: 260-8

23. Cowpe J, Plasschaert A, Harzer W, Vinkka-Puhakka H, Walmsley AD. Profile and competences for the graduating European dentist - update 2009. Eur J Dent Educ 2010; 14: 193-202

24. Hoffmeister L, Moya P, Vidal C, Benadof D. Factors associated with early childhood caries in Chile. Gac Sanit 2016; 30: 59-62

25. Soto L, Tapia, R., Rodriguez, G. Diagnóstico Nacional de Salud Bucal de Adolescente de 12 años y Evaluación del Grado de Cumplimiento de los Objetivos Sanitarios de Salud Bucal 2000-2010. Chile: MINSAL, 2007

26. Anderson P, Beeley J, Monteiro PM, de Soet H, Andrian S, Amaechi B, Huysmans MC. A European Core Curriculum in Cariology: the knowledge base. Eur J Dent Educ 2011; 15 Suppl 1: 18-22

27. Buchalla W, Wiegand A, Hall A. Decision-making and treatment with respect to surgical intervention in the context of a European Core Curriculum in Cariology. Eur J Dent Educ 2011; 15 Suppl 1: 40-4

28. Pitts N, Melo P, Martignon S, Ekstrand K, Ismail A. Caries risk assessment, diagnosis and synthesis in the context of a European Core Curriculum in Cariology. Eur J Dent Educ 2011; 15 Suppl 1: 23-31 